\title{
Remote Viewing with and without controlled Out-Of-Body Consciousness
}

\author{
Luciano Pederzoli*o, Elena Prati*॰, Arianna Ballati* and Patrizio Tressoldi* \\ ${ }^{\circ}$ EvanLab, Firenze, Italy
}

*Science of Consciousness Research Group, Dipartimento di Psicologia Generale, Università di Padova, Italy

\begin{abstract}
Four participants, chosen for their experience in reaching an Out-of-Body state of consciousness via hypnotic induction, were asked to describe five different and unknown locations, at first using Remote Viewing conditions in an ordinary state of consciousness and then again using Remote Viewing in Out-of-Body state of consciousness.

According to an assessment by two independent judges, the average percentages of information obtained in Remote Viewing were 55\% for those correct and 35\% for those wrong, while in Outof-Body they were $54 \%$ and $35 \%$ respectively.

The average percentage of identical information to both conditions was $14 \%$.

Furthermore, three out of four participants obtained a higher percentage of correct answers and a lower percentage of incorrect answers in the OB-RV condition.

This study illustrates the possibility of also using Remote Viewing in an Out-of-Body state of consciousness that is induced and controlled via hypnosis to obtain accurate information about unknown locations in an unconventional way.
\end{abstract}

Keywords: remote viewing, out-of-body experience, consciousness, hypnosis.

Corresponding author:

Patrizio Tressoldi

Email: patrizio.tressoldi@unipd.it 


\section{Introduction}

The main research hypothesis of this study, was to confirm if Remote Viewing (RV) in an hypnotically-induced Out-of-Body (OB) condition is more accurate in identifying target features than RV in an ordinary state of consciousness. The ability to obtain information from a distance about persons, events, physical places, objects and various types of buildings in an ordinary state of consciousness is commonly defined as RV. A comprehensive description of its characteristics, origins, techniques and range of applications is given by Stephan Schwartz (2017) and Russell Targ (2019).

In brief, this perception technique - in use since the 1960s - consists of asking someone to describe a specific and unknown target without any conventional means of acquiring information about the target itself.

Although the state of consciousness of people using this technique seems to resemble an ordinary wakeful state, the ability to differentiate the information about a specific target from that generated by normal mental activity, for example expected or inferred information, requires a special ability to filter this last one in order to identify the target's details, given that they don't travel through the sense organs.

The following is an example of how Joseph McMoneagle, one of the leading experts in $\mathrm{RV}$, describes his mental state:

"I find that when things work best for me is when I've been able to ample up my awareness about what's going on around me within specific circumstances. ... when I then verbalize what is going on inside me, my body, my head, my mind and physical properties, I have to in some way translate this into something understandable to another human being... I got a tremendous dose of impressions which contained a lot of noise, and a myriad of visual flashes, which were all nonsensical. In my efforts to try and decipher what was happening, I arrived at a purely hypothetical drawing of what tasted or felt the best to me... If anything, I'd have to say that I've learned to meditatively empty my mind and concentrate on choosing those things I need to pay attention to the most, and then being able to statistically derive an answer as to what I'm supposed to say. (Joe McMoneagle, personal communication).

From this short description it is clear that the effective use of RV requires having or reaching a specific mental state which is different to the ordinary waking state.

Experimental evidence obtained under strictly controlled conditions to eliminate any possibility of fraud or conventional access to information, supports the authenticity of this mental ability. The most recent quantitative synthesis of all studies conducted up to 2014, provided by Baptista, Derakhshani \& Tressoldi (2015), highlights an average accuracy percentage of $36.6 \%$ compared to an average percentage of $25 \%$ for random selections.

Even if an accuracy of $36.6 \%$ seems far from an ideal 100\%, under certain conditions and with very gifted participants promising practical results have been obtained in the recovery of archaeological finds (e.g. Schwartz, Mattei, \& The Moebius Society, 2000), in predicting financial events (e.g. Smith, Laham, \& Moddel, 2014), and in intelligence activities (e.g. Marwaha \& May, 2017; Targ, 2019). 


\section{Remote Viewing with Out-of-Body Consciousness}

In an OB state like that of induced with hypnotic suggestions is it possible to obtain information comparable to, or even better than, that obtained using RV?

Given that the OB state (see Alvarado, 2015, for a complete description of this unusual state of consciousness) has the peculiar trait of experiencing one's centre of consciousness as detached from the physical body, and therefore also from all sense organs, from the 1970s onwards some researchers have attempted to confirm the possibility of inducing a controlled state of $\mathrm{OB}$, and then asking participants to move their centre of consciousness to gather information about a specific target. The pioneers of this research path were Charles Tart and John Palmer.

For example, Palmer and Vassar (1974) attempted to induce OB experiences (OBEs) by progressive muscular relaxation techniques and audio-visual stimulation and then instructed their participants to travel to another room and to try to see a target. However, the subjects who reported OBEs obtained scores lower than would be obtained by chance alone. Palmer (1978), in summarizing his experiments, did not find support for the hypothesis that scores for subjects who report OBEs were statistically above chance and statistically higher than those of subjects who did not report OBEs.

A study in which OB was induced using hypnosis is described in Tart (1998). Seven participants, who were all in the upper $10 \%$ of the hypnotic susceptibility range, received a suggestion after they reached a very deep hypnotic state to move their consciousness to a distant locked laboratory and to carefully observe some specific target objects. However, none of their reports matched the characteristics of the targets and no formal analysis was completed.

Tressoldi \& Del Prete (2007) aimed at comparing the differences between hypnosis with an OB versus ordinary (no OB) hypnosis, with respect to the capacity to perceive distant pictures outside of the sensory visual range. In this study no differences were found between the two hypnotic inductions. In both conditions, participants obtained an overall accuracy of $3.7 \%$ above chance. Tressoldi et al. (2014) tried to replicate and improve the previous study of Tressoldi \& Del Prete (2007). Five participants selected for their experience with hypnotic inductions were induced into an $\mathrm{OB}$ and were requested to verbally describe six selected images placed in two different rooms located hundreds of kilometres from the place where they were under hypnosis. On average, the participants correctly identified $46.7 \%$ of the images, compared to a probability of $25 \%$ expected due to chance. However, this study did not have a control condition with which to compare the results obtained in the OB.

This study may be considered a conceptual replication of that of Tressoldi et al (2014).

The main differences are:

- in order to make it more interesting for the participants and the task more ecological, the targets were two churches, a museum and two historical buildings;

- all participants were asked to describe these targets at first in the RV condition, followed by the $\mathrm{RV}$ in the $\mathrm{OB}$ state (OB-RV).

\section{Method}

\section{Participants}

The participants were four women with an average age of 54, ranging from 42 to 69 , selected from the group of EvanLab candidates for their experience with the state of consciousness in OB induced 
via hypnotic suggestion. The experience varied from a minimum of 12 to a maximum of 37 sessions all conducted by the same hypnotist. However, none of them had ever experienced obtaining information from physical locations using either RV or OB-RV techniques. The hypnotist, LP, has been practicing hypnosis for research purposes for over 20 years and also has more than 10 years' experience in inducing non-ordinary states of consciousness.

\section{Procedure}

On the agreed day, each participant individually carried out an RV session followed by an OB$\mathrm{RV}$ induced via hypnotic suggestion, attempting to describe as accurately as possible the targets proposed by the hypnotist. Neither the hypnotist nor the participants had ever seen or visited the targets or the cities in which they were located.

When a participant signaled that she was ready, the hypnotist gave the following instructions: "Now concentrate and try to provide as much information as you can get on [target name] located in Treviso (city in Italy where all targets were located).

During the RV session the hypnotist's only task was to assist the participant to accurately describe all of the target's different features and find as many of them as possible.

At the end of the RV session, when the participant indicated being ready, the OB induction via hypnotic suggestion procedure began as described by Pederzoli \& Tressoldi (2018). Given that the participants were very familiar with this technique, the time required to subjectively reach the OB state varied from 4 minutes to a maximum of 7 minutes. When the participant replied in the affirmative to the question "Are you in an OB state?", she was asked to describe as accurately and completely as possible the same target as in the RV session.

In this session the hypnotist's task was identical to that in the RV session.

The decision to not alternate the order of RV and OB-RV sessions was made based on the hypothesis that in the latter it was easier to obtain more target information and thus it could influence the performance in RV if it was done immediately after. All the sessions were audio-recorded.

\section{Targets}

The targets were two churches (an old one and a contemporary one), a modern museum and two historical monuments in the city of Treviso, located in Italy's north-east, near Venice. This city and the targets within it were chosen because firstly, the hypnotist and participants were unfamiliar with it, and secondly, it was physically accessible so that afterwards the features described by participants could be checked and photos taken for data analyses. Photos of the five targets are available at https://doi.org/10.6084/m9.figshare.12192474.v3

\section{Data analyses}

The audio recordings of each session were sent to co-author PT, whose task was to compile a written list of every item of information provided by the four participants for each of the five targets. The only information not included were subjective opinions, such as: "There's a lovely entrance door", and anything that could not be verified, such as: "It's a place where initiation rites were held". 


\section{Scoring}

Each item of information provided by each participant was marked as either correct, partially correct (and reasons why), or wrong. Information which was totally correct or wrong was given a score of 1 point, and those partially correct were given half a point. Finally, all the points for these three categories were added for each target.

The same task was carried out by a second judge using the available photos, with no way of knowing if they refer to RV or OB-RV, such as the example shown in Table 1, with respect to target 'SV' presented in Figure 1:

Table 1: Example of evaluation relative to Target SV

\begin{tabular}{l|c|l}
\hline \multicolumn{1}{c|}{ Information } & YES/NO & \multicolumn{1}{c}{ PARTIAL } \\
\hline $\begin{array}{l}\text { A square-shaped bell tower no } \\
\text { taller than the church, }\end{array}$ & $\begin{array}{l}\text { Squared, but higher than the } \\
\text { church }\end{array}$ \\
\hline Ochre-coloured façade & YES & \\
\hline $\begin{array}{l}\text { Large door in the middle of } \\
\text { the entrance }\end{array}$ & YES & \\
\hline $\begin{array}{l}\text { A Romanic-type arch with } \\
\text { sculptures (the Good Shepherd } \\
\text { with sheep) }\end{array}$ & & $\begin{array}{l}\text { Not a Romanic-style arch, and } \\
\text { sculpture is not of the Good } \\
\text { Shepherd }\end{array}$ \\
\hline
\end{tabular}

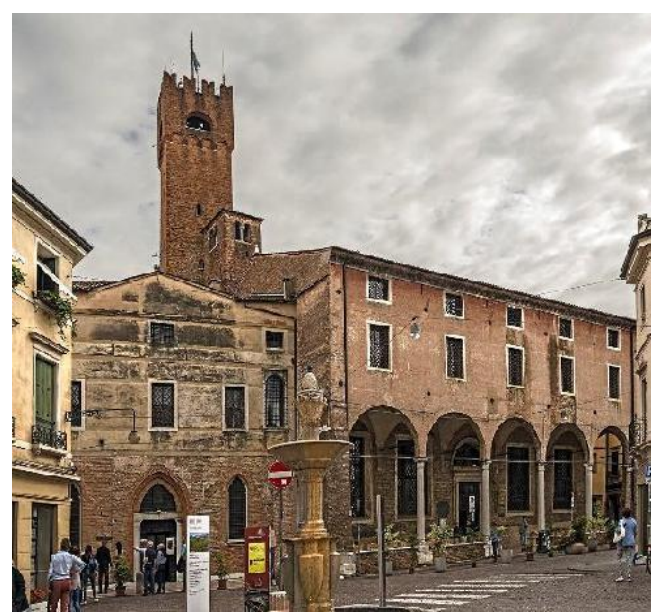

Figure 1: one of the pictures of target SV

Lists of the information relative to all $20 \mathrm{RV}$ and $20 \mathrm{OB}-\mathrm{RV}$ sessions, with their associated score given by two judges, are available at https://doi.org/10.6084/m9.figshare.12192474.v3 together with photos of the targets to allow independent verification. 


\section{Results}

The descriptive and inferential statistics relative to the information obtained from the $20 \mathrm{RV}$ and 20 OB-RV sessions are presented in Table 2, together with the percentage of identical information.

Table 2: Descriptive and inferential statistics of the number of information obtained in RV and in OB-RV and of the percentage of similar information in both conditions.

\begin{tabular}{c|c|c|c}
\hline & info in RV & info in OB-RV & \% Identical info \\
\hline Mean & 10.5 & 12.6 & .14 \\
\hline SD & 4.7 & 4.1 & .08 \\
\hline $\boldsymbol{p}^{*}$ & & .06 & \\
\hline \multicolumn{4}{|c}{$p^{*}=$ paired t-test $p$ value }
\end{tabular}

\section{Comment}

In the OB-RV condition, participants provided around two information more than in the RV one, but the most interesting finding is that only $14 \%$ of this information is identical to both the RV and OB-RV conditions (e.g. "Access stairway with 4 steps"; target LC; participant D).

Another relevant finding is that the participants very often obtained different information from the identical targets. The total number of different information is: $\mathrm{SP}=77 ; \mathrm{SV}=84 ; \mathrm{LC}=89$; $\mathrm{ME}=66 ; \mathrm{PSQ}=42$.

\section{Interjudges agreement}

The average Pearson correlation between the scores of the two judges was .71; 95\% CI: .39 .87; ranging from .84 for correct information in OB-RV condition to .44 for wrong information in the OB-RV condition.

The scoring of both judges is presented in the Supplementary Material section. It was therefore decided to use the average score given by the two judges to compare the descriptions between the RV and OB-RV conditions.

\section{Comparison between correct and wrong information}

Comparisons between correct and wrong information of all participants in the RV and OB-RV conditions are shown in Table 3 and Figures 2, 3, 4 and 5. Individual performances of participants are presented in Table S2 in the Supplementary Material.

Table 3: Descriptive and inferential statistics of the comparison between correct and wrong information obtained in RV and in OB-RV.

\begin{tabular}{c|c|c|c|c|c|c|c|c}
\hline & $\begin{array}{c}\text { Correct } \\
\text { info in } \\
\text { RV }\end{array}$ & $\begin{array}{c}\text { Wrong } \\
\text { info in } \\
\text { RV }\end{array}$ & $\begin{array}{c}\text { \% Corr. } \\
\text { iffo in } \\
\text { RV }\end{array}$ & $\begin{array}{c}\text { \% Wrong } \\
\text { info in } \\
\text { RV }\end{array}$ & $\begin{array}{c}\text { Correct } \\
\text { info in } \\
\text { OB-RV }\end{array}$ & $\begin{array}{c}\text { Wrong } \\
\text { info in } \\
\text { OB-RV }\end{array}$ & $\begin{array}{c}\text { \% Corr } \\
\text { info in } \\
\text { OB-RV }\end{array}$ & $\begin{array}{c}\text { \% Wrong } \\
\text { info in } \\
\text { OB-RV }\end{array}$ \\
\hline Mean & 5.5 & 4.05 & .54 & .36 & 6.9 & 4.2 & .55 & .34 \\
\hline SD & 2.3 & 2.3 & .10 & .11 & 2.7 & 1.8 & .09 & .10 \\
\hline $\boldsymbol{E S d}$ & & .98 & & .86 & & .99 & & 1.08 \\
\hline $\boldsymbol{p}^{*}$ & & .0001 & & .0001 & & .0001 & & .0001 \\
\hline
\end{tabular}

$\mathrm{ES} d=$ Cohen's d effect size; $p^{*}=$ paired t-test $p$ value 


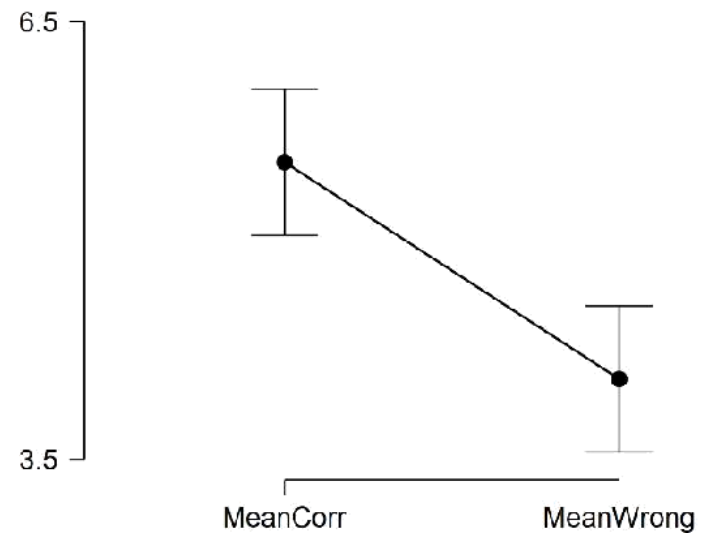

Figure 2: Means and 95\% confidence intervals related to the comparison between the number of correct and wrong information in the RV condition.

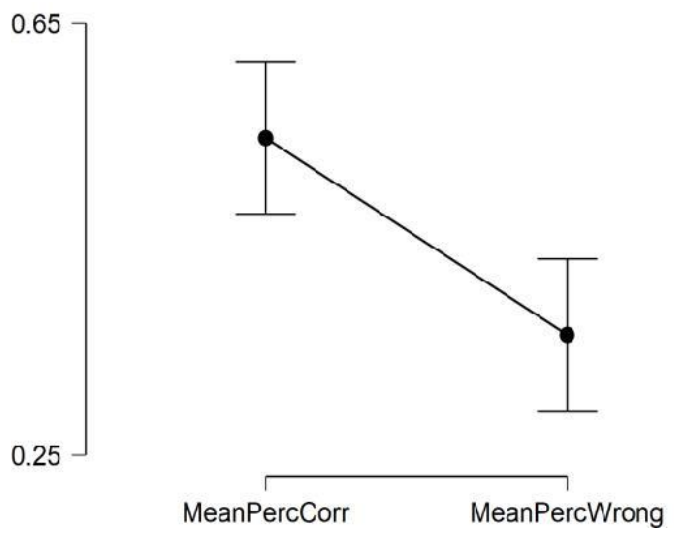

Figure 3: Means and 95\% confidence intervals related to the comparison between the percentages of correct and wrong information in the RV condition.

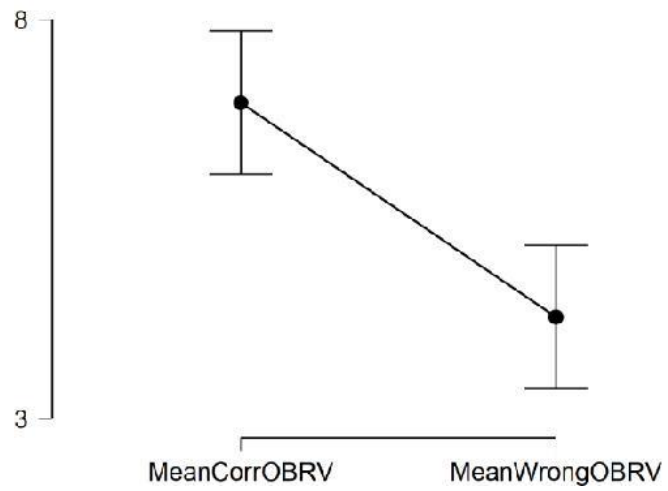

Figure 4: Means and 95\% confidence intervals related to the comparison between the number of correct and wrong information in the OB-RV condition 


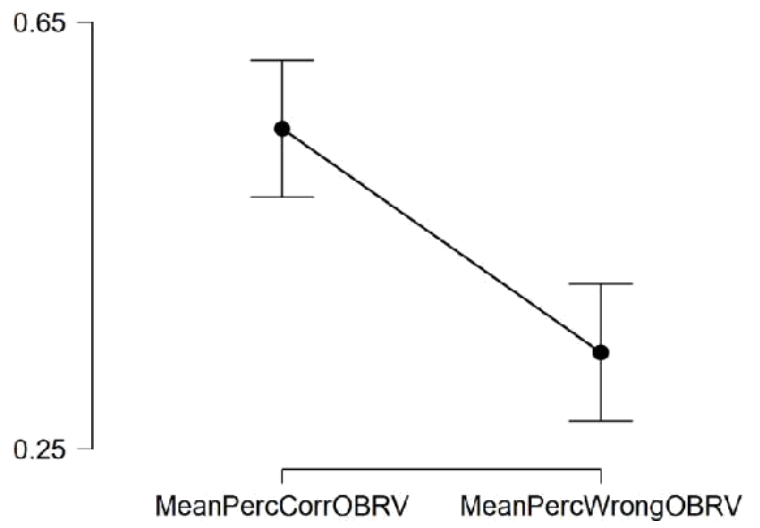

Figure 5: Means and 95\% confidence intervals related to the comparison between the percentages of correct and wrong information in the OB-RV condition.

\section{Comment}

The differences between the number and the percentage of correct and wrong information both in RV and OB-RV clearly show the correct ones to be greater.

In percentage terms, this difference is around $20 \%$ in both conditions. The total of percentages of correct and wrong answers is not equal to 100 because partially correct answers were given a score of half a point.

\section{Comparison between RV and OB-RV performances}

Table 4 shows this comparison.

Table 4: Descriptive and inferential statistics related to the comparison between correct and wrong information obtained in the RV and in the OB-RV conditions.

\begin{tabular}{c|c|c|c|c|c|c|c|c}
\hline & $\begin{array}{c}\text { Correct } \\
\text { info in } \\
\text { RV }\end{array}$ & $\begin{array}{c}\text { Correct } \\
\text { info in } \\
\text { OB-RV }\end{array}$ & $\begin{array}{c}\text { Wrong } \\
\text { info in } \\
\text { RV }\end{array}$ & $\begin{array}{c}\text { Wrong } \\
\text { info in } \\
\text { OB-RV }\end{array}$ & $\begin{array}{c}\text { \% Corr } \\
\text { info in } \\
\text { RV }\end{array}$ & $\begin{array}{c}\text { \% Corr } \\
\text { info in } \\
\text { OB-RV }\end{array}$ & $\begin{array}{c}\text { \% Wrong } \\
\text { info in } \\
\text { RV }\end{array}$ & $\begin{array}{c}\text { \% Wrong } \\
\text { info in } \\
\text { OB-RV }\end{array}$ \\
\hline Mean & 5.5 & 6.9 & 4.05 & 4.2 & .54 & .55 & .36 & .34 \\
\hline SD & 2.3 & 2.7 & 2.3 & 1.9 & .10 & .09 & .11 & .10 \\
\hline $\boldsymbol{E S d}$ & & .50 & & .09 & & .05 & & .16 \\
\hline $\boldsymbol{p}^{*}$ & & .04 & & .69 & & .81 & & .47 \\
\hline
\end{tabular}

\section{Comment}

The only difference between RV and OB-RV conditions is in the raw number of correct information, resulting in an average of one more information in the OB-RV condition.

However, if the individual performance of the four participants, presented in Table S2 in the Supplentary Material, is analyzed, it can be observed that three out of four participants had a higher percentage of correct answers and a lower percentage of incorrect answers in the OB-RV condition. 


\section{Discussion}

The purpose of this study was to compare the ability to identify and describe physical targets, from a distance, in the RV and OB-RV states of consciousness.

The results clearly demonstrate that in both conditions, the amount of correct information is clearly greater than wrong information, with a difference of around $20 \%$. The only difference in performance between the two is in the number of correct information, which is slightly greater in the OB-RV condition.

An interesting finding from this comparison is that the percentage of identical information to both the RV and OB-RV is only around $14 \%$.

This fact not only suggests that in these two states of consciousness the participants were able to gather different information, but also that they did not simply add the information obtained in RV to that reported in OB-RV.

We remind that our choice not to alternate the two conditions, derived from the hypothesis that information obtained in OB-RV - which we believed to be more favorable - could be used in the RV if it was used afterwards.

Furthermore, this finding also suggests that the participants were not influenced by an expectation of OB-RV being the more favorable, with a consequent reduction of effort and quality of performance during the RV.

However, in the hindsight, a better experimental design is to use different targets in the RV and OB-RV conditions within each session. For example, session 1: target A for the RV condition and target B for the OB-RV; session 2 (after one week): target A for the OB-RV condition and target B for the RV condition. This experimental design variant, doesn't exclude the possibility that participants can remember the information obtained the week before, but it increases the possibility to forget it.

\section{Possible sources of fraud}

The possibility of the participants acquiring the relevant information through conventional means can be excluded. Indeed, apart from there being no doubt about their integrity from an ethical point of view, they could not have known the target in advance of the experiment and each participant did not know about the others, thus ruling out any information exchange.

The only real potential source of fraud or influence could have been the hypnotist, who knew about the target before the participants did and could have given them information during the sessions, possibly favoring OB-RV. Other than the fact that the hypnotist's professional integrity is beyond question, many of the target's details were not available on the internet and could only have been obtained by actually visiting the sites or by access to the photos used by the judges, but which were, however, taken after the experiment had ended.

With respect to the judges, even though one is a co-author (PT), the other judge was totally unaware of what type of experiment this was. Furthermore, as already stated, all photos and information provided by the participants are freely available at https://doi.org/10.6084/m9.figshare.12192474.v3 for independent verification. 


\section{Conclusions}

Despite its limitations, this work indicates that it's possible to use the OB state of consciousness to obtain information from a distance in a way that supports the well-known RV techniques.

The efficacy of these procedures is sufficient for practical applications, obviously using qualified participants. For example, as it results from the total number of different information gathered by the four participants, it is possible to obtain a large number of details of the same target for its better identification. 
Acknowledgements: we thank Cinzia Evangelista for English translation and revision and Stephan Schwartz for comments and suggestions. We also thank our participants for their voluntary and motivated participation. 


\section{References}

Alvarado, C. S. (2015). Out-of-Body Experience (OBE). In Psi Encyclopedia. London: The Society for Psychical Research. https://psi-encyclopedia.spr.ac.uk/articles/out-bodyexperience-obe . Retrieved 10 April 2020.

Marwaha, S. B., \& May, E. (2017). The Star Gate archives: reports of the US government sponsored PSI program -1972-1995. An overview. In Paper Presented at the 60th Annual Convention of the Parapsychological Association (pp. 1-21).

Palmer, J. (1978). "ESP and out-of-body experiences: an experimental approach. In Mind Beyond the Body, ed D. S. Rogo (New York, NY: Penguin Books).

Palmer, J., and Vassar, C. (1974). ESP and out-of-the-body experiences: an exploratory study. Journal of American Society for Psychical Research, 68, 257-280.

Pederzoli, L., \& Tressoldi, P. E. (2018). A Guide for OBE Induction. SSRN Electronic Journal. https://doi.org/10.2139/ssrn.3148432

Schwartz, S. (2017). 'Remote Viewing'. Psi Encyclopedia. London: The Society for Psychical Research. https://psi-encyclopedia.spr.ac.uk/articles/remote-viewin>. Retrieved 10 April 2020

Schwartz, S. A., Mattei, R. J. De, \& Society, T. M. (2000). The discovery of an American brig: fieldwork involving applied remote viewing including a comparison with electronic remote sensing. Archaeology, 73-78.

Smith, C. C., Laham, D., \& Moddel, J. (2014). Stock market prediction using associative remote viewing by inexperienced remote viewers. Journal of Scientific Exploration, 28(1), 7-16.

Targ, R. (2019). What Do We Know about Psi? The First Decade of Remote-Viewing Research and Operations at Stanford Research Institute. Journal of Scientifi c Exploration, 33(3), 569-592.

Tressoldi, P. E., Pederzoli, L., Caini, P., Ferrini, A., Melloni, S., Richeldi, D., ... Trabucco, A. (2014). Out of Body Experience Induced by Hypnotic Suggestion. Part 1: Phenomenology and Perceptual Characteristics. SSRN Electronic Journal. https://doi.org/10.2139/ssrn.2443719

Tart (1998). Six studies of out-of-body experiences. Journal of Near Death Studies, 17, 73-99. doi: 10.1023/A:1022932505993

Tressoldi, P. \& Del Prete, G. (2007). ESP under hypnosis: the role of induction instructions and personality characteristics. Journal of Parapsychology, 71, 125-137. 


\section{Supplementary Material}

Table S1a: Descriptive statistics of the correct and wrong information obtained in RV and in OB-RV, calculated by Judge 1

\begin{tabular}{c|c|c|c|c|c|c|c|c}
\hline & $\begin{array}{c}\text { Correct } \\
\text { info in } \\
\text { RV }\end{array}$ & $\begin{array}{c}\text { Wrong } \\
\text { info in } \\
\text { RV }\end{array}$ & $\begin{array}{c}\text { \% Correct } \\
\text { info in } \\
\text { RV }\end{array}$ & $\begin{array}{c}\text { \% Wrong } \\
\text { info in } \\
\text { RV }\end{array}$ & $\begin{array}{c}\text { Correct } \\
\text { info in } \\
\text { OB-RV }\end{array}$ & $\begin{array}{c}\text { Wrong } \\
\text { info in } \\
\text { OB-RV }\end{array}$ & $\begin{array}{c}\text { \% Correct } \\
\text { info in } \\
\text { OB-RV }\end{array}$ & $\begin{array}{c}\text { \% Wrong } \\
\text { info in } \\
\text { OB-RV }\end{array}$ \\
\hline Mean & 6.1 & 4.1 & .61 & .36 & 8.1 & 3.7 & .64 & .29 \\
\hline SD & 2.6 & 2.6 & .16 & .16 & 3.4 & 2.2 & .14 & .15 \\
\hline
\end{tabular}

Table S1b: Descriptive statistics of the correct and wrong information obtained in RV and in OB-RV, calculated by Judge 2

\begin{tabular}{c|c|c|c|c|c|c|c|c}
\hline & $\begin{array}{c}\text { Correct } \\
\text { info in } \\
\text { RV }\end{array}$ & $\begin{array}{c}\text { Wrong } \\
\text { info in } \\
\text { RV }\end{array}$ & $\begin{array}{c}\text { \% Correct } \\
\text { info in } \\
\text { RV }\end{array}$ & $\begin{array}{c}\text { \% Wrong } \\
\text { info in } \\
\text { RV }\end{array}$ & $\begin{array}{c}\text { Correct } \\
\text { info in } \\
\text { OB-RV }\end{array}$ & $\begin{array}{c}\text { Wrong } \\
\text { info in } \\
\text { OB-RV }\end{array}$ & $\begin{array}{c}\text { Correct } \\
\text { info in } \\
\text { OB-RV }\end{array}$ & $\begin{array}{c}\text { \% Wrong } \\
\text { info in } \\
\text { OB-RV }\end{array}$ \\
\hline Mean & 4.9 & 4.0 & .48 & .36 & 5.7 & 4.8 & .46 & .38 \\
\hline SD & 2.3 & 2.5 & .13 & .16 & 2.2 & 2.2 & .08 & .12 \\
\hline
\end{tabular}

Table S2: Descriptive statistics of the percentages of correct and wrong information of each single participant.

\begin{tabular}{cccccc}
\hline & ID & \%CorrRV & \%WrongRV & \%CorrOBRV & \%WrongOBRV \\
\hline Mean & D & 0.508 & 0.395 & 0.544 & 0.340 \\
SD & & 0.060 & 0.078 & 0.124 & 0.139 \\
\hline Mean & E & 0.503 & 0.386 & 0.523 & 0.355 \\
SD & & 0.069 & 0.051 & 0.095 & 0.093 \\
\hline Mean & $\mathrm{N}$ & 0.590 & 0.320 & 0.544 & 0.369 \\
SD & & 0.119 & 0.162 & 0.057 & 0.083 \\
\hline Mean & $\mathrm{V}$ & 0.575 & 0.345 & 0.589 & 0.298 \\
SD & & 0.146 & 0.149 & 0.109 & 0.126 \\
\hline
\end{tabular}

